\title{
Coronavirus Disease 2019 Outbreak: Use of a Chest X-ray Scoring System and Evaluation of the Radiologic Findings
}

\author{
Aslı Tanrıvermiş Sayit' (D), Çetin Çelenk' $\mathbb{D}$, Ş eyma Genç² (D) \\ 'Department of Radiology, Ondokuz Mayıs University Faculty of Medicine, Samsun, Turkey \\ ${ }^{2}$ Department of Public Health, Ondokuz Mayıs University Faculty of Medicine, Samsun, Turkey \\ ORCID iDs of the authors: A.T.S. 0000-0003-286I-I56X; Ç.Ç. 0000-0002-6078-7525; S.G. 0000-0002-2885-584X.
}

Cite this article as: Sayit AT, Çelenk Ç, Genc S. Coronavirus disease 2019 outbreak: Use of a Chest X-ray Scoring System and Evaluation of the Radiologic Findings. Cyprus J Med Sci. 2021; 6(4): 325-330.

\section{BACKGOUND/AIMS}

Our objective was to identify the chest X-ray (CXR) features of the patients with coronavirus disease 2019 (COVID-19) and to evaluate the relationship between CXR scores and age and gender. Also, we aimed to detect the sensitivity, positive predictive value (PPV), and negative predictive value (NPV) of CXRs in patients with COVID-19 pneumonia.

\section{MATERIAL and METHODS}

A total of 35 patients who underwent CXR and computed tomography (CT) and had a positive real-time reverse transcriptasepolymerase chain reaction test result were included in the study. The initial CXRs of all patients were evaluated and scored using the Brixia scoring system. Then, chest CT scans were assessed the presence of pneumonia.

\section{RESULTS}

Of the 35 patients, CXRs of 13 patients ( 6 male and 7 female) were normal, and 22 patients had unilateral or bilateral opacities, which was considered to indicate pneumonia. The sensitivity, PPV, NPV, and accuracy of the CXRs in the detection of pneumonia were $81.4 \%$, $100 \%, 61.5 \%$, and $85.7 \%$, respectively. The median Brixia score of patients without pneumonia was less than that of the patients with pneumonia (4 [I-6] vs $7[4-12] ; P<.01)$.

\section{CONCLUSION}

This scoring system might be useful for identifying the highest-risk patients at an early stage and determine who requires early medical management in the pandemic service or intensive care unit.

Keywords: SARS-CoV-2, COVID-19, chest radiography, pneumonia, BRIXA score

\section{INTRODUCTION}

Severe acute respiratory syndrome coronavirus 2 (SARS-CoV-2) is a novel coronavirus that was first detected in Wuhan, Hubei Province of China, in December 2019.' The disease was named as coronavirus disease 2019 (COVID-19) and affected a large number of people in a short time. COVID-19 disease was announced as a global pandemic on March II, 2020 by the World Health Organization. ${ }^{2}$ By January 30, 202I, more than 100 million cases and 2 million deaths have been reported around the world. ${ }^{3}$

The precise diagnostic test for COVID-19 disease is a reverse transcription-polymerase chain reaction (RT-PCR) assay. ${ }^{4}$ However, the RT-PCR results may be negative due to an inadequate nasopharyngeal or oropharyngeal swab sample. In addition, the RT-PCR results are usually available within I-2 days. Therefore, radiological imaging methods have become critical in the early detection and treatment, especially patients with suspected pneumonia. Although its sensitivity to detect pneumonia is not very high, chest radiography is the first radiological imaging method that should be performed, especially children and young adults with suspected pneumonia. The most commonly reported chest X-ray (CXR) features of the COVID-19 pneumonia are bilateral pulmonary infiltration and consolidation, often involving the lower lobes. ${ }^{5}$ Wong et al. ${ }^{6}$ conducted a study including 64 patients that evaluated the frequency and pattern of CXR features and reported the sensitivity of CXR in the detection of SARS-CoV-2 pneumonia, which was 69\% [95\% Cl: 56-80\%]. Evaluating 
the CXR can be confusing, especially in patients with chronic disease such as heart failure and chronic lung disease. The Brixia scoring system, which is a semiquantitative scoring method, was established to evaluate the severity of COVID-19 on a CXR due to the high virulence and mortality of the disease, especially in patients with comorbid diseases, such as hypertension, diabetes mellitus, and cardiovascular and oncological diseases. ${ }^{7}$ This scoring system might help to determine who is under high risk and who require specific treatment modality.

Due to the close relationship between pulmonary involvement and mortality, we aimed to (i) explain the CXR findings in patients with SARS-CoV-2 infection who had a positive RTPCR test finding, (ii) evaluate the relationship between CXR scores and age and gender, and (iii) determine the sensitivity, positive predictive value (PPV), and negative predictive value (NPV) of CXR in patients with SARS-CoV-2 pneumonia.

\section{MATERIAL and METHODS}

This retrospective study was approved by the institutional review board of our hospital, and a written informed consent was waived due to the outbreak of COVID-19 disease (2020/ 450).

\section{Patients}

Between March II and June 10, 2020, we identified I00 patients who had a positive nasopharynx or oropharynx RT-PCR test result and admitted to the emergency service with complaints of fever, dyspnea, dry cough, and weakness. A total of 35 patients who underwent CXR and chest CT were included in the study. Of these 35 patients, 15 (42.9\%) were female, and 20 (57.1\%) were male. The age, gender, medical history, and presence of comorbidities were retrospectively evaluated with data from the electronic medical record of our hospital. This is a blinded study. The initial CXRs of all patients were evaluated and scored by one radiologist (Ç.Ç.) experienced in thoracic radiology. Then, the other radiologist (A.T.S.) reviewed all chest CT scans for the presence of pneumonia.

\section{CXR Scoring System}

Posteroanterior (PA) CXRs were obtained by having the patient stand in the upright position with full inspiration. Anteroposterior (AP) CXRs were acquired in patients who were unable to stand when using a mobile chest radiograph machine. All CXRs were acquired as computed or digital radiographs following usual local protocols in the emergency department. Sometimes, CXRs may have relatively low quality due to emergency service circumstances. All isolation rules

\section{Main Points}

- SARS-CoV-2 is a newly identified $\beta$-coronavirus that leads to an outbreak of unusual viral pneumonia.

- The precise diagnostic test for COVID-19 disease is a RTPCR test.

- In the presence of suspicion of pneumonia, CXR should be done first.

- The BRIXA scoring system can be used in patients with infiltration on CXR, which can estimate the course of the disease and the prognosis of the patient. against contamination of a possible infection were obeyed in the radiology unit. The radiologist described the presence, localization, and characterization of infiltrations in the PA or AP radiographs without the knowledge of the $C T$ findings.

We used the Brixia scoring system, which was defined by Borghesi and Maroldi, to score the patients' CXRs. ${ }^{4}$ We ranked pulmonary involvement on an I8-point severity scale according to the extent and characteristics of lung abnormalities.

The Brixia scoring system includes two steps. In the first step, the lungs are divided into six regions as follows:

Upper zones (A and D): above the inferior wall of the aortic arch.

Middle zones ( $B$ and $E$ ): below the inferior wall of the aortic arch and above the inferior wall of the right inferior pulmonary vein.

Lower zones ( $\mathrm{C}$ and $\mathrm{F}$ ): below the inferior wall of the right inferior pulmonary vein. ${ }^{4}$

In the second step, we gave point (from 0 to 3) for each side based on lung abnormalities identified on PA or AP radiographs as follows:

- Score 0: no lung abnormalities

- Score I: interstitial infiltrates

- Score 2: interstitial and alveolar infiltrates (interstitial predominance)

- Score 3: interstitial and alveolar infiltrates (alveolar predominance $)^{4}$

Then, all scores of the six regions were summed to obtain a total score between 0 and 18. After defining and scoring the CXR findings, chest CT scans of all patients were assessed for the presence of pneumonia.

\section{CT Imaging}

Noncontrast-enhanced chest CT scans (Canon, Aquilion Prime SP, Canon Medical Systems, Japan) were performed for patients who were evaluated with CXRs who had a pathology on their CXR or a normal CXR with suspected pneumonia during physical examination. All the chest $\mathrm{CT}$ scans acquired as a noncontrast-enhanced volumetric scans with a low dose technique (tube voltage, $120 \mathrm{kV}$; tube current, standard (reference $\mathrm{mAs}, 60-120$ ) to low dose (reference $\mathrm{mAs}, 30$ ) with automatic exposure control; slice thickness, $1.0 \mathrm{~mm}$ ) in the emergency department, which was specifically separated in the isolation area. Chest CT was performed in a supine position and full inspirium. They described the presence, localization, shape, density, and borders of all lesions. The lesions were defined as patchy, confluent, or nodular according to their shape. Patchy lesions were non-nodular isolated focal lesions, and confluent lesions were large lesions with multiple segment involvement that tended to merge with each other. The pattern of lesions from patchy to confluent was classified as pure ground-glass opacity (GGO), mixed GGO and consolidation, consolidation, and crazy-paving pattern. The density of nodular lesions was defined as pure GGO, part-solid, and solid. The 
Table I. Demographic Findings of the Patients

\begin{tabular}{|c|c|c|c|c|}
\hline \multicolumn{2}{|c|}{ Age (years) } & \multirow{2}{*}{$\begin{array}{c}\text { Female }(n=15) \\
\begin{array}{c}45.8 \pm 24.2 \\
1\end{array}\end{array}$} & \multirow{2}{*}{$\begin{array}{c}\text { Male }(n=20) \\
46.05 \pm 19 \\
5\end{array}$} & \multirow[t]{2}{*}{$\begin{array}{r}P \\
.98\end{array}$} \\
\hline Symptoms & Fever & & & \\
\hline & Cough & 10 & 8 & \\
\hline & Dyspnea & 0 & 4 & \\
\hline & Asymptomatic & 2 & 2 & \\
\hline Treatment & Pandemic service & 13 & 14 & \\
\hline
\end{tabular}

\begin{tabular}{|lcc|}
\hline Table 2. Comorbidities of the Female and & Male Patients & \\
\hline Comorbidities & Female, $\mathbf{n}$ & Male, $\mathbf{n}$ \\
\hline Asthma & 1 & 0 \\
Hypertension & 3 & 2 \\
Diabetes mellitus type II & 3 & 0 \\
Medical history of previous carcinoma & 4 & 2 \\
Coronary artery disease & $\mathrm{I}$ & 2 \\
Hyperlipidemia & $\mathrm{I}$ & 1 \\
Chronic renal disease & 0 & 1 \\
Chronic obstructive lung disease & 0 & 1 \\
Cerebrovascular disease & $\mathrm{I}$ & 1 \\
\hline
\end{tabular}

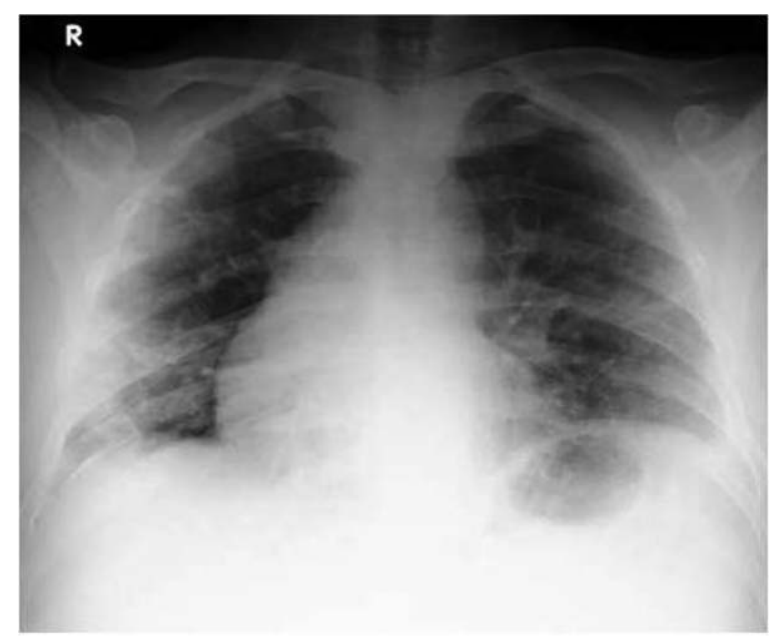

Figure I. A chest X-ray (CXR) showed multifocal patchy peripheral consolidations in the middle and lower zones of both lungs, especially in the right lung. The Brixia score of the CXR was 14. (The right lung score was 7 , and the left lung score was 7.)

borders of the infiltrations were described as well-demarcated or ill-demarcated. In addition, the presence of an air bronchogram, a reverse halo, and a tree-in-bud appearance was described. ${ }^{8}$

\section{Statistical Analysis}

Statistical Package for the Social Sciences (SPSS) version 21 (IBM SPSS Corp.; Armonk, NY, USA) was used to perform all statistical calculations. The normality of distribution for the variables was examined using the Shapiro-Wilk test. Descriptive analysis results were expressed as the mean and standard deviation for normally distributed variables. The median (minimum-maximum) was used for non-normally distributed varia-

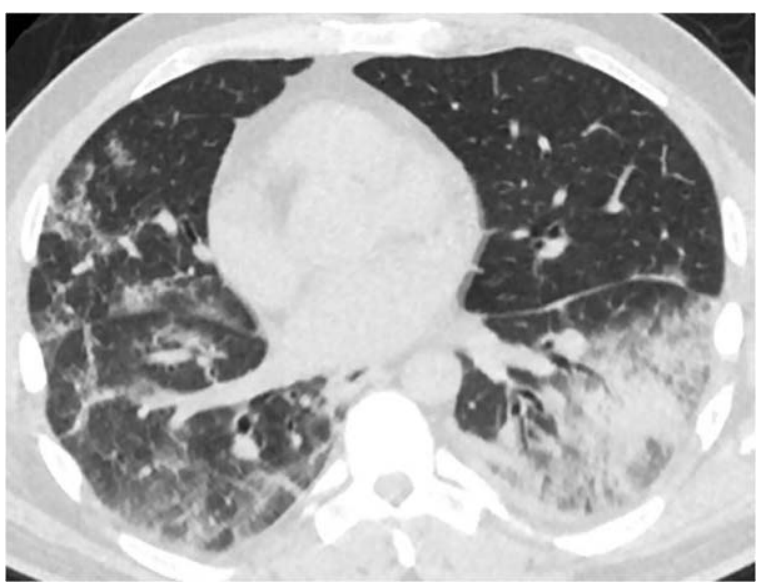

Figure 2. An axial nonenhanced chest computed tomography (CT) image showed patchy ground-glass opacities (GGOs) in the right lung with consolidative lesions in the left lower lobe.

bles. Categorical variables are expressed as percentages. The student t-test was used for normally distributed variables, and the Mann-Whitney $U$ test was used for non-normally distributed variables. The sensitivity, specificity, PPV, NPV, and accuracy of the CXR were compared to reference test, which was chest CT. A value of $P<.05$ was considered significant in all statistical analyses.

\section{RESULTS}

\section{Patients}

Of the 35 patients $(45.97 \pm 2$ lyears), 15 were female $(45.87 \pm$ 24.27 years), and 20 were male ( $46.05 \pm 19.01$ years) (Table I). No statistically significant differences were detected in age between the female and male patients $(P=.98)$. Of the 35 patients, $27(77.1 \%)$ were treated in the pandemic service and discharged from the hospital without any complications. Eight patients $(22.9 \%)$ received intensive care. Three of them died, and five of them were discharged from the hospital. The age of the patients who received intensive care $(65.7 \pm 16.9$ years $)$ was significantly higher than that of those treated in the pandemic service (40.II \pm 18.6 years) $(P=.001)$. The comorbidities of the patients are shown in Table 2 .

\section{CXR Features and Scoring System}

The CXRs of 13 patients were normal ( 6 males and 7 females), and the CXRs of 22 patients demonstrated unilateral or bilateral opacities ( 15 males and 7 females), which indicated pneumonia. The CXR interval of patients was $3 \pm 2.5$ days during the 


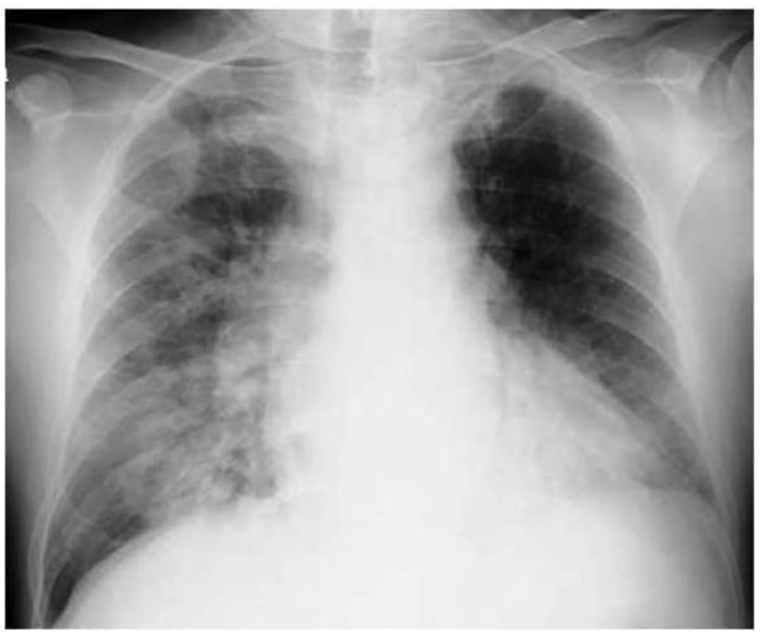

Figure 3. A CXR showed multifocal patchy consolidations in the upper, middle, and lower zones of the right lung and slightly increased opacity in the left lower zone. The Brixia score of the CXR was 16. (The right lung score was 12 , and the left lung score was 4.)

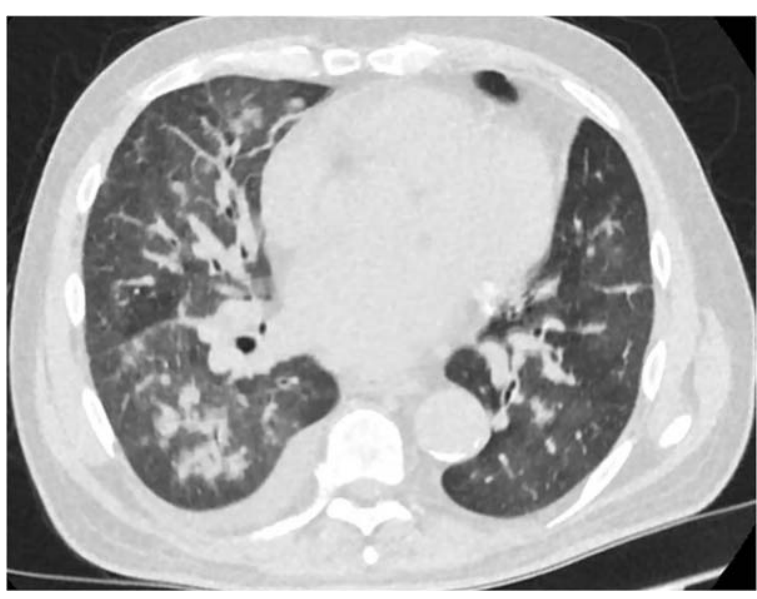

Figure 4. An axial nonenhanced chest $\mathrm{CT}$ showed slight GGOs in both lungs with rounded GGOs in the right lung. first admission before CT examination. The sensitivity, PPV, NPV, and accuracy of chest radiography in the detection of pneumonia were $81.4 \%, 100 \%, 61.5 \%$, and $85.7 \%$, respectively. The Brixia score in the CXR reports ranged from I to 12 . The patients were classified into two groups with and without pneumonia as regards to CXR results. The Brixia score of patients without pneumonia was $4(\mathrm{I}-6)$, and the Brixia score of those with pneumonia was 7 (4-12) (Figures I and 2). There was a statistically significant difference in the Brixia scores between patients with and without pneumonia $(P<.01)$ (Table 3$)$. The Brixia score of the 27 patients who were treated with medication in the pandemic service was $5(|-| \mid)$, and the Brixia score of the eight patients who were treated in the intensive care unit was $8(5-12)(P=.004)$ (Figures 3 and 4 and Table 3$)$.

\section{Chest X-ray Correlation with CT}

The low-dose chest CT results of all patients were evaluated for the presence of pneumonia. The mean radiation dose was $2.22 \pm 1.85 \mathrm{mSv}$. Of the 35 patients, eight $(24.5 \pm 18.4$ years $)$ had normal thorax CT images, and 27 (52.3 \pm 17.4 years) had characteristic features of COVID-19 pneumonia. Of these 27 patients, three had unilateral (right) pneumonia, and $24 \mathrm{had}$ bilateral lung parenchymal abnormalities, which were considered SARS-CoV-2 pneumonia. The chest CT imaging features are shown in Table 4. When the chest CT scans and CXRs were retrospectively evaluated, opacities were visualized on chest CT but not CXRs in five of the 27 patients (Figures 4 and 5). The CXRs of these patients were reevaluated for the Brixia score by dividing them into two groups as patients with or without pneumonia according to chest CT features. The Brixia score of patients with pneumonia (6 [1-2]) was significantly higher than those without pneumonia $(2[I-6])(P=0.001)$.

\section{DISCUSSION}

In here, the Brixia score of patients with pneumonia was significantly higher than the patients without pneumonia. Also, the sensitivity, PPV, and accuracy of the CXRs in the detection of pneumonia were found high in our study. CXR is primarily the preferred imaging method for evaluating the pneumonia due to its wide availability and low radiation exposure. Also, it is the least expensive imaging method. However, the sensitivity of CXRs in the diagnosis of pneumonia is not as high as that of CT. They may lead to relatively low quality of the images due to emergency service circumstances. CXRs may be more useful

Table 3. Per-Lesion Analysis of Chest X-Ray and Computed Tomography (CT) Findings

\section{Chest X-Ray Findings}

Laterality

Density

Pneumonia

BRIXA score

BRIXA score

BRIXA score
Rightlung 8

Left lung

Bilateral 13

Normal chest X-ray 13

Consolidation

Diffüz opacity 33

Patients with pneumonia $\quad 22$

Normal chest X-ray

Patients with pneumonia $7(4-12)$

Normal chest X-ray $4(1-6)$

Patients who were treated in the pandemic service $\quad 5(I-I I)$

Patients who were treated in the intensive care unit $8(5-12)$

Patients with pneumonia in the thorax CT $6(1-12)$

Patients with normal thorax CT 


\section{Table 4. Imaging Characteristic on the Thorax CT in 27 Patients}

\section{Opacity Characteristics}

Peripheral multifocal ground-glass opacities

Multifocal rounded ground glass opacities

"Crazy-paving" pattern

Alveolar consolidation with ground glass opacities

"Reverse halo" sign

Airways

Bronchial wall thickening

Bronciectasis

Underlying Lung Disease

Pulmonary emphysema

Pulmonary fibrosis

Other Findings

Pleural effusion

Pericardial effusion

Atelectasis

Thoracic lympadenopathy

Abbreviation: CT: computed tomography.

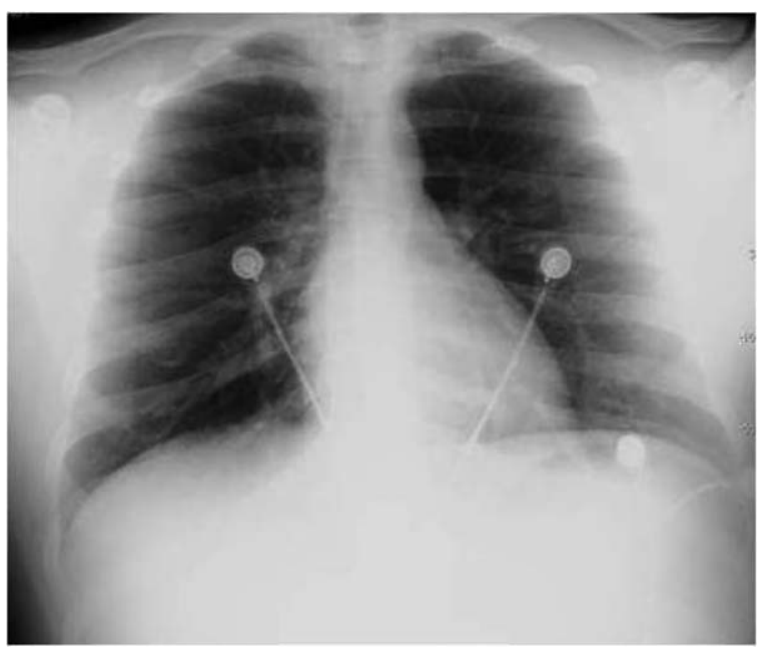

Figure 5. The CXR was normal. There was no pneumonic infiltration or pleural effusion. The Brixia score of the CXR was 4. (The right lung score was 2, and the left lung score was 2.)

and sensitive in the diagnosis of pneumonia in children and young adults.' The BRIXA scoring system might help identify the highest-risk patients at an early stage and determine who requires early medical management in the pandemic service or intensive care unit.

Recent studies have shown that the distribution of CXR features and lung abnormalities shows a variable appearance. ${ }^{6}$ During the SARS-CoV-2 outbreak in 2003, bilateral disease and infiltration of more than two zones were reported on CXRs and related to worse outcomes. ${ }^{9,10}$ Similar imaging findings have been identified in various other pneumonias. ${ }^{11,12}$ Although CXR is generally accepted as the reference standard, a recent Cochrane review identified two studies that suggested that routine CXR did not affect outcomes in patients with lower respiratory tract infections. ${ }^{13}$

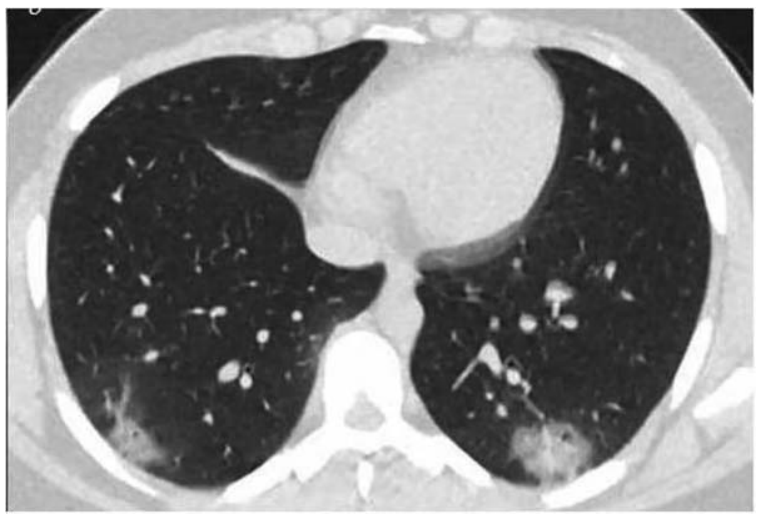

Figure 6. An axial nonenhanced chest CT image showed rounded GGOs in the peripheral regions of the lower lobes.

Wong et al. ${ }^{6}$ revealed that the sensitivity of an initial RT-PCR analysis (91\% [95\% Cl: 83-97\%]) was higher than that of a baseline CXR (69\% [95\% Cl: 56-80\%]) $(P=.009)$. They also reported that six patients showed CXR abnormalities before eventually testing positive with RT-PCR. In their study, all patients with positive CXRs also had positive thorax $C T$, and only one patient $(I / 4,25 \%)$ had a falsely negative CXR when compared to thorax $\mathrm{CT}^{6}$. In our study, the sensitivity, PPV, NPV, and accuracy of chest radiography were $81.4 \%, 100 \%, 61.5 \%$, and $85.7 \%$, respectively, for detecting COVID-19 pneumonia. All patients with positive CXRs also had positive chest $C T$, and only five patients had a false negative CXR when made a comparison with chest CT.

The most frequent CXR and CT features of SARS-CoV-2 pneumonia are bilateral, peripheral, and basilar opacities/densities.' However, CXRs can be normal in some patients. There may be two reasons for this situation. First, GGOs may not have been detected on the CXR, which may not have had sufficient density (Figure 6). Second, lesions located in basal and retrocardiac areas are very difficult to evaluate. ${ }^{14}$ In our study, 22 of the 35 patients had increased opacity on their CXRs, which indicated pneumonia, and a positive RT-PCR test. Of the 35 patients, 13 had normal CXRs. However, five of the 13 patients had GGOs on chest CT, which was considered pneumonia. Peripheral and basal zone distribution were the most frequent locations, and most had bilateral involvement, which is compatible with the findings reported in the literature. There was bilateral involvement in 13 patients, right lung involvement in eight patients, and left lung involvement in one patient. However, when the chest CT scans of these patients were evaluated, only three patients had right lung involvement, while 24 had bilateral involvement. The other chest CT scans were normal.

Toussie et al.' evaluated the prognostic value of a CXR severity scoring system for younger (2l-50-year-old) patients with COVID-19. CXRs were divided into three zones per lung, and severity score was scored according to the presence (I point) or absence ( 0 point) of opacity in each region. They reported that a CXR score $\geq 3$ was an independent predictor of intubation $(n=28)(P=.002)$ at the hospital site. They also reported no significant difference in primary outcomes across race/ethnicity, history of tobacco use, asthma, or diabetes mellitus type II.' 
Borghesi et al. ${ }^{7}$ reported that the CXR score was significantly higher in males than in females only in those aged $50-79$ years. They showed that males aged $\geq 50$ years and females aged $\geq 80$ years with SARS-CoV-2 infection had the highest CXR score (median $\geq 8$ ). They concluded that the Brixia score can help identify the highest-risk patients at an early stage and identify who needs specific treatment and who needs protection from the infection. ${ }^{7}$

In our study, the Brixia score was significantly higher in patients with pneumonia than in patients without pneumonia. Also, Brixia score was significantly higher in patients who were treated in the intensive care unit than those treated in the pandemic service.

Using the Brixia score is easy and quick for showing the severity index of lung infiltration. It can assist early diagnosis, treatment, and supplies the relevant information about lung infiltration. CXR is an inexpensive, low-cost, easily accessible, and low-radiation imaging method to evaluate lung abnormalities and is especially preferred in children and younger adults. According to the Fleischner Society, chest CT is not recommended for patients who have mild symptoms or are asymptomatic. ${ }^{15,16}$ Therefore, chest CT should be performed in patients who had a suspected moderate-to-severe clinical features of the SARS-CoV-2 infection, additional comorbidities, or opacities on a CXR. In addition, patients with features of pneumonia on thorax CT can be followed up with a CXR because of its low radiation dose and low rate of infectiousness due to its portability. However, in patients with a high Brixia score based on the CXR and suspected progression of pneumonia, chest CT should be performed to evaluate the lung parenchyma. In addition, the use of low-dose CT scans was recently started to prevent patients from receiving high doses of radiation. In our study, the lowest radiation dose was $0.15 \mathrm{mSv}$, and the highest radiation dose was $7.67 \mathrm{mSv}$.

Small number of patients is the limitation of our study. The reason for the small sample size is that most patients with suspected pneumonia directly undergo chest CT without an initial CXR. Studies with a larger sample of patients can contribute to the literature. Since no false positive case for CXRs, it is not possible to calculate specificity. So, not to have specificity result is the another limitation.

The Brixia score was significantly higher in patients with pneumonia than in patients without pneumonia. Also, it was significantly higher in patients who were treated in the intensive care unit than those treated in the pandemic service. This scoring system might contribute to identification of the highest-risk patients at an early stage and determination of who requires early medical management in the pandemic service or intensive care unit.

Ethics Committee Approval: The ethical approval was obtained from the Ethical Committee of Ondokuz Mayis University Faculty of Medicine $(2020 / 450)$.

Informed Consent: Written informed consent was obtained from all participants who participated in this study.

Peer-review: Externally peer-reviewed.
Author Contributions: Concept - A.T.S., Ç.Ç., S.G.; Design - A.T.S.; Supervision - A.T.S., Ç.Ç., S.G.; Resources - A.T.S.; Materials - A.T.S., S.G.; Data Collection and/or Processing - A.T.S., Ç.C.., S.G.; Analysis and/or Interpretation - A.T.S.; Literature Search - A.T.S., Ç.Ç.; Writing Manuscript A.T.S.; Critical Review - A.T.S.

Conflict of Interest: The authors have no conflicts of interest to declare.

Financial Disclosure: The authors declared that this study has received no financial support.

\section{REFERENCES}

I. Toussie D, Voutsinas N, Finkelstein M, et al. Clinical and chest radiography features determine patient outcomes in young and middle age adults with COVID-19. Radiology. 2020;297(I):E197-E206. [CrossRef]

2. Li Q, Guan X, Wu P, et al. Early transmission dynamics in Wuhan, China, of novel coronavirus-infected pneumonia. N Engl J Med. 2020;382:1|99-1207. [CrossRef]

3. COVID-19 Map. Johns Hopkins coronavirus resource center. 2016. Available at https:/ / coronavirus.jhu.edu./map.htlm.

4. Borghesi A, Maroldi R. COVID-19 outbreak in Italy: Experimental chest $X$-ray scoring system for quantifying and monitoring disease progression. Radiol Med. 2020;125:509-5I3. [CrossRef]

5. Chen SG, Chen JY, Yang YP, et al. Use of radiographic features in COVID-19 diagnosis: Challenges and perspectives. J Chin Med Assoc. 2020;83:644-647. [CrossRef]

6. Wong HYF, Lam HYS, Fong AH, et al. Frequency and distribution of chest radiographic findings in patients positive for COVID-19. Radiology. 2020;296(2):E72-E78. [CrossRef]

7. Borghesi A, Zigliani A, Masciullo R, et al. Radiographic severity index in COVID-19 pneumonia: Relationship to age and sex in 783 Italian patients. Radiol Med. 2020;125(5):46I-464. [CrossRef]

8. Hansell DM, Bankier AA, MacMahon H, McLoud TC, Muller NL, Remy J. Fleischner society: Glossary of terms for thoracic imaging. Radiology. 2008;246(3):697-722. [CrossRef]

9. Hui DS, Wong KT, Antonio GE, et al. Severe acute respiratory syndrome: Correlation between clinical outcome and radiologic features. Radiology. 2004;233(2):579-585. [CrossRef]

10. Antonio GE, Wong KT, Tsui EL, et al. Chest radiograph scores as potential prognostic indicators in severe acute respiratory syndrome (SARS). AJR. 2005;|84(3):734-74I. [CrossRef]

II. Lim WS, Macfarlane JT. Defining prognostic factors in the elderly with community acquired pneumonia: A case controlled study of patients aged $\geq 75$ yrs. Eur Respir J. 2001;17:200-205. [CrossRef]

12. Leroy O, Devos P, Guery B, et al. Simplified prediction rule for prognosis of patients with severe community-acquired pneumonia in ICUs. Chest. 1999;116(I):157-165. [CrossRef]

13. Cao AMY, Choy JP, Mohanakrishnan LN, Bain RF, van Driel ML. Chest radiographs for acute lower respiratory tract infections. Cochrane Database Syst Rev. 2013;2013(12):CD009119.

14. Li B, Li X, Wang Y, et al. Diagnostic value and key features of computed tomography in coronavirus disease 2019. Emerg Microbes Infect. 2020;9(I):787-793. [CrossRef]

15. Rubin GD, Ryerson CJ, Haramati LB, et al. The role of chest imaging in patient management during the COVID-19 pandemic: A multinational consensus statement from the fleischner society. Chest. 2020;158(I):I06-116. [CrossRef]

16. Rubin GD, Ryerson CJ, Haramati LB, et al. The role of chest imaging in patient management during the COVID-19 pandemic: A multinational consensus statement from the fleischner society. Radiology. 2020;296(I):172-180. [CrossRef] 